\title{
Metal-Organic Nanosheets Formed via Defect-Mediated Transformation of a Hafnium Metal-Organic Framework
}

Matthew J. Cliffe, ${ }^{\dagger}$ († Elizabeth Castillo-Martínez, ${ }^{\dagger}$ (i) Yue Wu, ${ }^{\dagger}$ Jeongjae Lee, ${ }^{\dagger}$ Alexander C. Forse, ${ }^{\dagger}$ Francesca C. N. Firth, ${ }^{\dagger}$ Peyman Z. Moghadam, ${ }^{\S}$ David Fairen-Jimenez, ${ }^{\S}$ Michael W. Gaultois, ${ }^{\dagger}$ Joshua A. Hill," Oxana V. Magdysyuk, ${ }^{\perp}$ Ben Slater, ${ }^{\#}$ Andrew L. Goodwin," and Clare P. Grey ${ }^{*} \dagger$

${ }^{\dagger}$ Department of Chemistry, University of Cambridge, Lensfield Road, Cambridge CB2 1EW, U.K.

${ }^{\ddagger}$ Department of Materials Science \& Metallurgy, University of Cambridge, 27 Charles Babbage Road, Cambridge CB3 0FS, U.K.

${ }^{\S}$ Department of Chemical Engineering and Biotechnology, University of Cambridge, Pembroke Street, Cambridge CB2 3RA, U.K.

"Department of Chemistry, University of Oxford, South Parks Road, Oxford OX1 3QR, U.K.

${ }^{\perp}$ Diamond Light Source Ltd., Harwell Science and Innovation Campus, Didcot, Oxfordshire OX11 0DE, U.K.

\#Department of Chemistry, University College London, 20 Gordon Street, London WC1H 0AJ, U.K.

\section{Supporting Information}

ABSTRACT: We report a hafnium-containing MOF, hcp $\mathrm{UiO}-67(\mathrm{Hf})$, which is a ligand-deficient layered analogue of the face-centered cubic fcu UiO-67(Hf). hcp UiO-67 accommodates its lower ligand:metal ratio compared to fcu UiO-67 through a new structural mechanism: the formation of a condensed "double cluster" $\left(\mathrm{Hf}_{12} \mathrm{O}_{8}(\mathrm{OH})_{14}\right)$, analogous to the condensation of coordination polyhedra in oxide frameworks. In oxide frameworks, variable stoichiometry can lead to more complex defect structures, e.g., crystallographic shear planes or modules with differing compositions, which can be the source of further chemical reactivity; likewise, the layered hcp UiO-67 can react further to reversibly form a two-

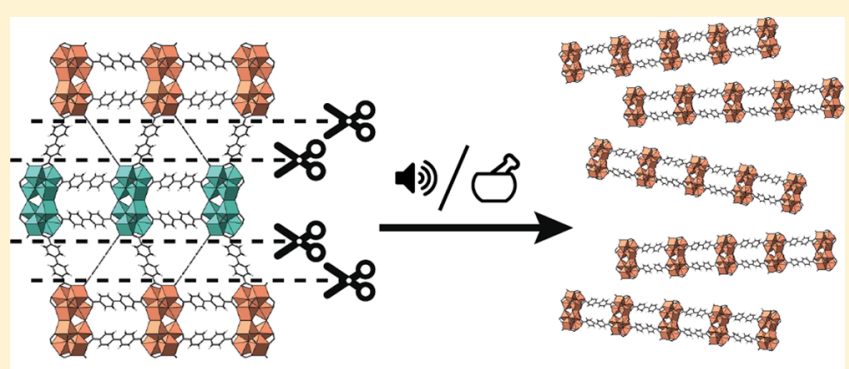

hcp UiO-67 nanosheets dimensional metal-organic framework, hxl UiO-67. Both three-dimensional hcp UiO-67 and two-dimensional hxl UiO-67 can be delaminated to form metal-organic nanosheets. Delamination of hcp UiO-67 occurs through the cleavage of strong hafniumcarboxylate bonds and is effected under mild conditions, suggesting that defect-ordered MOFs could be a productive route to porous two-dimensional materials.

\section{INTRODUCTION}

In many inorganic functional materials, compositional flexibility is facilitated not just by inclusion of vacancies but also through the formation of higher-dimensionality defects, such as stacking faults, dislocations, or crystallographic shear planes. The presence of one- or two-dimensional features, and the resultant consequences for microstructure, can in many materials be the most important factor in controlling the mechanical properties (e.g., dislocations in metals control plastic deformation) or thermal properties (e.g., reducing thermal conductivity through phonon scattering). ${ }^{1,2}$ Although the mechanisms through which these higher-dimensional defects are accommodated at a local level vary among materials, one common feature (especially in oxides) is the condensation of coordination polyhedra, e.g., the conversion of corner-sharing vanadium oxide polyhedra into edge-sharing polyhedra in the Magnéli phases to accommodate the oxide:metal ratio., ${ }^{3,4}$ The process of defect accommodation creates new sites with differing chemical reactivities. For example, the layered Dion-Jacobson or Ruddlesden-Popper families of defect perovskites can often be delaminated to create free-standing two-dimensional (2D) nanosheets that can be then used as, e.g., photocatalysts. ${ }^{5-7}$

Metal-organic frameworks (MOFs) are framework materials that consist of nodes of metal atoms or clusters linked together by organic molecular ligands. They are materials of great current interest, in particular because of their unique and tailorable porosities, which facilitate applications as wideranging as gas separation and storage, selective catalysis, and drug delivery. Although defects and nonstoichiometry have been shown to be a critical factor in the chemistry of MOFs, both as a determinant of structure and as a source of useful functionality, ${ }^{8,9}$ this field is still in its infancy. To date, along with multicomponent $\mathrm{MOFs},{ }^{10}$ particular attention has been paid to the preparation and characterization of materials containing point defects, principally ligand and metal-cluster vacancies. ${ }^{8,11}$ These vacancies not only reduce the overall density of the material (thus increasing the porosity) but also

Received: January 4, 2017

Published: March 27, 2017 
introduce reactive sites that can be the source of catalytic activity. ${ }^{12}$ At present, three main routes have been developed for the synthesis of MOFs containing high concentrations of ligand-absence defects, i.e., missing ligands: extensive postsynthesis washing (which can remove soluble ligands) ${ }^{13}$ the "ligand-fragmentation" approach, where a ligand without the full complement of binding sites is included in the synthesis mixture, e.g., where a dicarboxylic acid is introduced into a tricarboxylic acid MOF; $;^{10,14}$ and, most importantly, the "modulator" approach. ${ }^{11,12}$

Modulated synthesis, in which relatively large quantities of nonbridging monotopic acids (e.g., acetic or benzoic acid) are added to the reaction mixture (as many as 250 molar equivalents relative to the ligand), was originally introduced as a method for controlling the morphology of MOF particles ${ }^{15,16}$ but has since proven to be a very productive route to a wide variety of ligand-deficient MOFs. ${ }^{8,12}$ It has been particularly successful for group 4 MOFs, where the resultant vacancies can reach concentrations of up to $45 \%{ }^{13}$ The identity of the modulator is a critical factor for the resultant MOF in terms of both the concentration of defects-modulators of greater acidity tend to introduce larger numbers of vacancies ${ }^{13}$ - and also their arrangement: control over the wide variety of defect phases found for tetracarboxylic acid group 4 MOFs can be achieved through judicious choice of modulating acid. ${ }^{17-20}$ In previous work we found that the use of very high concentrations of formic acid in the synthesis of UiO-66 (the prototypical member of the group 4 MOF family, assembled from $\mathrm{Zr}_{6} \mathrm{O}_{4}(\mathrm{OH})_{4}$ clusters and terephthalic acid) not only introduced high concentrations of vacancies but also led to the formation of nanodomains of a lower-connectivity reo topology framework, containing cluster absences, within the ordinary defective framework. ${ }^{21,22}$ In all of these examples, the effect of the reduced ligand:metal ratio caused by the modulator in the reaction mixture was to introduce ligand or cluster point vacancies, whether distributed randomly or in ordered domains, rather than to form higher-dimensional defect structures.

Here we show that using the modulator approach to control the ligand:metal ratio enables us to synthesize a new Hfcontaining MOF, hcp UiO-67, in which ligand-deficiency is accommodated by the condensation of the hafnium oxide nodes to form a double cluster $\left(\mathrm{Hf}_{12} \mathrm{O}_{8}(\mathrm{OH})_{14}\right)$, in an analogous manner to variable-stoichiometry oxides. We explore the conditions under which this material forms, making use of in situ X-ray diffraction measurements under synthetic conditions, and its subsequent reactivity, demonstrating that it slowly converts to a $2 \mathrm{D}$ crystalline metal-organic material, hxl UiO-67, consisting of stacked 2D metal-organic sheets. We further show that both hcp and hxl UiO-67 can be delaminated into metal-organic nanosheets, demonstrating a new route to porous $2 \mathrm{D}$ materials, and characterize these new phases and explain their reactivity using a combination of powder X-ray diffraction (PXRD), transmission electron microscopy (TEM), pair distribution function (PDF) analysis, and quantumchemical calculations.

Two-dimensional materials are currently a topic of particular interest across a wide range of applications, perhaps most notably for the unique electronic properties of graphene and other electronic materials ${ }^{24-26}$ but also as catalysts and separation membranes because of the enhanced accessibility of and diffusion through low-dimensional materials. ${ }^{27-29}$ Metal-organic nanosheets have shown great promise as electronic sensors, ${ }^{30}$ gas sorption membranes, ${ }^{31}$ and catalysts, ${ }^{32}$ but the range of metal-organic nanosheets is currently dramatically more constrained than for three-dimensional (3D) MOFs. Most current synthesis has focused on three routes: ${ }^{33}$ assembly at an interface, ${ }^{30,34}$ synthesis of nanosheets in bulk solution, ${ }^{31,32}$ and liquid-phase exfoliation of weakly bound 2D layered materials (including with the assistance of surfactants). ${ }^{35,36}$ To the best of our knowledge, there have been no reports of the synthesis of nanosheets from 3D framework materials, as this requires the breaking of strong metal-ligand bonds. This work, by showing the feasibility of chemically selective cleavage of these strong bonds, suggests that harnessing the huge variety of 3D MOFs as precursors for metal-organic nanosheets is a promising strategy for the synthesis of new low-dimensional materials.

\section{METHODS}

Synthesis of hcp UiO-67. Biphenyl-4,4'-dicarboxylic acid $\left(\mathrm{H}_{2} \mathrm{bpdc}\right)(72.3 \mathrm{mg}, 0.3 \mathrm{mmol})$ and $\mathrm{HfCl}_{4}(96.1 \mathrm{mg}, 0.3 \mathrm{mmol})$ were added to a $23 \mathrm{~mL}$ PTFE-lined steel autoclave, followed by $4 \mathrm{~mL}$ of $\mathrm{N}, \mathrm{N}$-dimethylformamide (DMF) and then $1 \mathrm{~mL}$ of formic acid. The autoclave was sealed and heated to $150{ }^{\circ} \mathrm{C}$ for $24 \mathrm{~h}$. The resultant white microcrystalline powder of single-phase hcp UiO-67 was filtered under vacuum and washed on the filter with approximately $5 \mathrm{~mL}$ of DMF. Samples were activated using a two-step method adapted from ref 13. First, unreacted ligand was removed by washing with DMF at $70{ }^{\circ} \mathrm{C}$ for $24 \mathrm{~h}$, and then residual DMF was removed by one of two methods: either the DMF was exchanged through repeated washing with chloroform $(3 \times 10 \mathrm{~mL})$ followed by heating at $150{ }^{\circ} \mathrm{C}$ for $24 \mathrm{~h}$ (used to prepare the sample for adsorption measurements) or the DMF was removed by heating at $200{ }^{\circ} \mathrm{C}$ for $24 \mathrm{~h}$. Analysis calculated for $\mathrm{C}_{252} \mathrm{H}_{172} \mathrm{Hf}_{24} \mathrm{O}_{116}: \mathrm{C}, 32.2 ; \mathrm{H}, 1.9 ; \mathrm{N}, 0.0$. Found: C, 32.2; H, 1.7; $\mathrm{N}, 0.0$.

Reversible Formation of hxl UiO-67. As-synthesized samples of hcp UiO-67 were left under ambient conditions for 1 week, leading to complete conversion to hxl UiO-67. Small quantities of crystalline $\mathrm{H}_{2}$ bpdc were detectable in the PXRD patterns. A $50 \mathrm{mg}$ sample of hxl UiO-67/ $\mathrm{H}_{2}$ bpdc formed in this way was heated in $20 \mathrm{~mL}$ of DMF at $70{ }^{\circ} \mathrm{C}$ for $24 \mathrm{~h}$, and the resulting mixture was filtered under vacuum, yielding a white microcrystalline powder that was confirmed to be hcp UiO-67 by PXRD.

Delamination. A $50 \mathrm{mg}$ sample of hcp UiO-67 was suspended in $20 \mathrm{~mL}$ of methanol. The suspension was then sonicated for $30 \mathrm{~min}$ and left to settle for $24 \mathrm{~h}$. Evaporation of the supernatant after a further 5 days of sedimentation ( 6 days total) showed that stable suspensions of approximately $0.1 \mathrm{mg} \mathrm{mL} \mathrm{m}^{-1}$ could be obtained (the yield from $10 \mathrm{mg}$ of hcp UiO-67 in $10 \mathrm{~mL}$ of $\mathrm{MeOH}$ was $10 \%$ ). Samples for further analysis (microscopy and PXRD measurements) were obtained from both the supernatant solution and the precipitate. PXRD and TEM measurements on the supernatant and the settled powder confirmed that both had been delaminated. An equivalent procedure was followed for hxl UiO-67.

Powder X-ray Diffraction. All samples were assessed for crystallinity and purity via their PXRD patterns, which were measured using a PANalytical Empyrean diffractometer $(\mathrm{Cu} \mathrm{K} \alpha$ radiation, $\lambda=$ $1.541 \AA$ ) over the $2 \theta$ range $3-40^{\circ}$ using a step size of $0.02^{\circ}$ and a scan speed of $0.02^{\circ} \mathrm{s}^{-1}$. Additional measurements on hxl UiO-67 and hcp UiO-67 were carried out at beamline I11 at the Diamond Light Source using an X-ray energies of $15.0171 \mathrm{keV}(\lambda=0.82562 \AA)$ and 15.0071 $\operatorname{keV}(\lambda=0.826168 \AA)$, respectively. ${ }^{37,38}$ Analysis of all powder diffraction data (including indexing, Pawley refinement, and Rietveld refinement) was carried out using the TOPAS-Academic 4.1 structure refinement software. ${ }^{39-41}$

Structure Solution. Structure solution was carried out in three steps. First, the PXRD patterns were indexed, and a Pawley refinement was carried out to obtain accurate peak intensities and peak shape and instrumental broadening parameters. With the parameters obtained from Pawley refinement kept fixed, simulated annealing was carried 
(a)

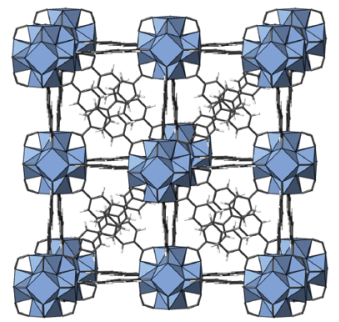

(d)

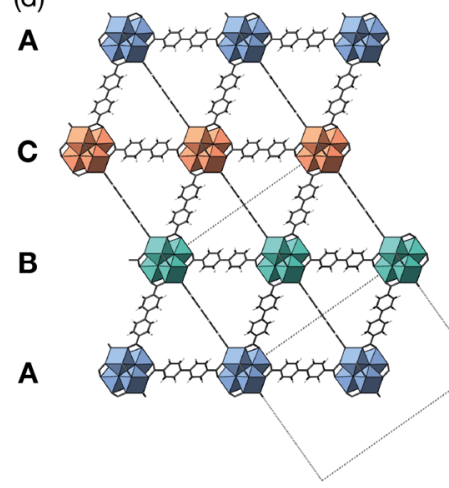

(g)

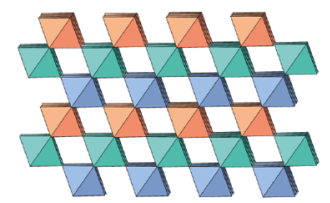

(b)

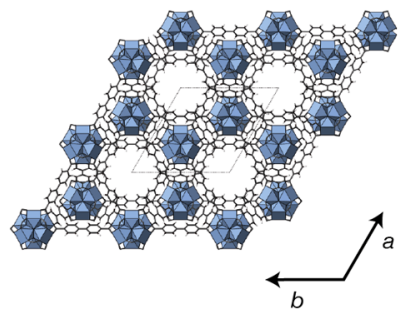

(e)

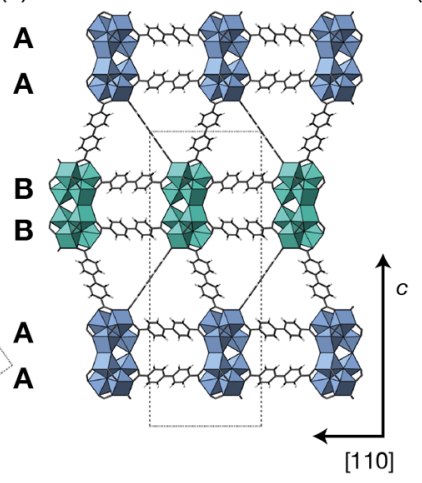

(h)

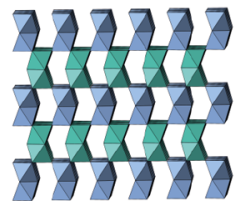

(c)

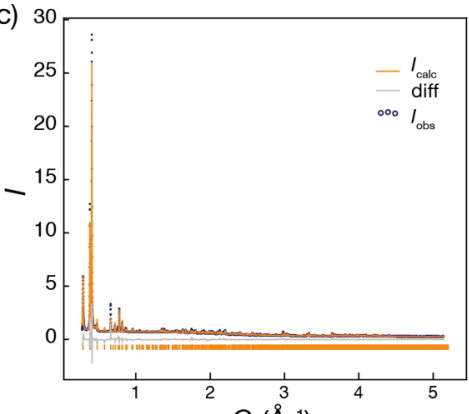

(f)

$Q\left(\AA^{-1}\right)$

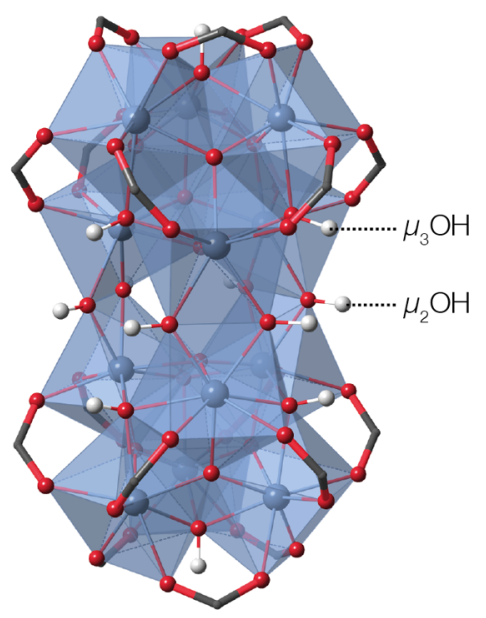

Figure 1. Crystal structure of hcp UiO-67 and its relationship to fcu UiO-67. (a) fcu UiO-67 viewed along the [001] direction. ${ }^{23}$ (b) hcp UiO-67 viewed along the [001] direction. (c) Rietveld refinement with synchrotron PXRD data for activated hcp UiO-67. (d) fcu UiO-67 viewed along the $[10 \overline{1}]$ direction highlighting the "ABC" stacking sequence. (e) hcp UiO-67 viewed along the [100] direction, highlighting the "ABBA" stacking sequence. (f) View of the double cluster in hcp UiO-67. (g) Cubic " $3 \mathrm{C}$ " perovskite $\mathrm{BaTiO}_{3}$ viewed along the $[10 \overline{1}]$ direction. (h) Hexagonal "4H" perovskite $\mathrm{CaMnO}_{3}$ viewed along the $[100]$ direction. The structures are represented as coordination polyhedra, with $\mathrm{HfO}_{8}$ polyhedra shown in blue, in (a) and (b). Color scheme: Hf, blue; O, red; H, white; C, black. In panels (d), (e), (g), and (h), the stacking sequence is highlighted by coloring the coordination polyhedra according to their position within the $a b$ plane: those in the "A" position are colored blue, those in the " $\mathrm{B}$ " position are colored green, and those in the " $\mathrm{C}$ " position are colored orange. The A-site cations have been omitted from panels ( $\mathrm{g}$ ) and $(\mathrm{h})$ for clarity.

out with $\mathrm{Hf}_{6}$ clusters allowed to freely rotate and translate throughout the cell. Simulated annealing runs with different cell occupancies were carried out until a structure that reproduced the general features of the experimental diffraction data was obtained. From these approximate models it was possible to build reasonable structure models, from which the symmetry could be obtained using the FINDSYM program. ${ }^{42}$ These symmetrized experimental models were then optimized using density functional theory (DFT) both with and without the full crystal symmetry. The validity of these computational models was verified by Rietveld refinement against synchrotron PXRD data, where the positions of $\mathrm{Hf}$ atoms were allowed to freely refine (see Supporting Discussion 3 for further details).

In Situ Synthesis Diffraction Measurements. The in situ XRD experiments were performed at beamline I12 at the Diamond Light Source using the ODISC furnace in the solvothermal configuration. ${ }^{43,44}$ Data were collected using a Thales Pixium image plate detector $(430 \mathrm{~mm} \times 430 \mathrm{~mm})$ with $12 \mathrm{~s}$ exposures. The $2 \mathrm{D}$ data were integrated and converted to conventional 1D PXRD data using DAWN. ${ }^{45}$ The energy of the monochromatized beam was $55.414 \mathrm{keV}$ ( $\lambda=0.22374 \AA$ ). The synthesis of hcp UiO-67 was carried out as described above, with a few minor adaptations for the in situ conditions: the reaction was carried out in a $5 \mathrm{~mL}$ culture tube, so the total volume of reactants was reduced from 5 to $3.5 \mathrm{~mL}$ (with all concentrations kept the same). The reaction mixture was also stirred with a PTFE bead to ensure that the measured volume of the reaction was representative of the reaction mixture as a whole.

Pair Distribution Function Analysis. Total scattering X-ray diffraction patterns were collected on beamline I15 at the Diamond Light Source using an X-ray energy of $72.0 \mathrm{keV}(\lambda=0.1722 \AA)$. Samples were packed in 0.0485 in. diameter Kapton capillaries (ColeParmer) and sealed with wax. Data were integrated and standard corrections applied using the DAWN software package. ${ }^{45}$ These raw data were further corrected (for background and Compton scattering) and Fourier transformed using PDFGetX3 ${ }^{46}$ The corrections were also carried out using GudrunX as a check on the consistency of the processed PDF data. ${ }^{47}$ Structural models were calculated using PDFGui from the optimized model of hcp UiO-67 and from the experimental crystal structure of fcu UiO-67 reported by Øien et al., ${ }^{23}$ lowered to $P 1$ symmetry to allow for the elimination of partial occupancies. $Q_{\text {damp }}$ was set to $0.1 \AA^{-1}$, and the isotropic displacement parameter $\left(U_{\text {iso }}\right)$ was $0.05 \AA^{2}$ for $\mathrm{C}, \mathrm{H}$, and $\mathrm{O}$ and $0.005 \AA^{2}$ for Hf.

Transmission Electron Microscopy. For TEM studies, a drop of the methanolic supernatant solution and another drop of the precipitate in solution were independently diluted in methanol and dispersed. A drop of this suspension was evaporated on a copper grid coated with holey carbon. Selected-area electron diffraction (SAED) and high-resolution TEM (HRTEM) were performed using a JEOL JEM-3011 electron microscope operated at $250 \mathrm{kV}$ under lowillumination conditions and equipped with a double-tilt $\pm 20^{\circ}$ sample 
holder; Gatan Digital Micrograph software was used to acquire images and perform further image processing. The presence of $\mathrm{Hf}$ was confirmed by energy-dispersive X-ray spectroscopy (EDX).

Quantum-Chemical Calculations. Ab initio DFT calculations were performed on the pristine, vacancy-free structures using the CASTEP code. $^{48}$ The non-spin-polarized Perdew-Burke-Ernzerhof (PBE) exchange-correlation functional ${ }^{49}$ was used with a $500 \mathrm{eV}$ plane-wave energy cutoff (corresponding to the "medium" setting in CASTEP). Because of the large cell size and hexagonal symmetry, reciprocal space was sampled only at the $\Gamma$ point. Electronic selfconsistent field cycles were converged to $10^{-4} \mathrm{eV}$. Structural relaxation was performed with convergence criteria of $10^{-3} \mathrm{eV}$ for the total energy and $0.05 \mathrm{eV} \AA^{-1}$ for the force. The cell was relaxed by first only allowing the atomic positions to relax, followed by the cell parameters.

Additional DFT calculations to probe the energetics of substituting $\mathrm{bpdc}^{2-}$ for formate in both intraplane and interplane positions $\left(\Delta E_{c}-\right.$ $\Delta E_{a b}$ ) were performed using the Quickstep module in CP2 $\mathrm{K}^{50}$ (www. cp2k.org). For consistency, the non-spin-polarized PBE exchangecorrelation functional ${ }^{49}$ was used. In the defect calculations, a double- $\zeta$ plus polarization basis ${ }^{51}$ and a cutoff of $850 \mathrm{Ry}$ were used. Selfconsistent field cycles were converged to $10^{-6} \mathrm{eV}$ and forces on atoms to $0.03 \mathrm{eV} \AA^{-1}$ or less.

\section{RESULTS AND DISCUSSION}

Inspired by the unusual behavior of formic acid as a modulator in other group $4 \mathrm{MOFs}^{22}$ we investigated its effect on the synthesis of UiO-67, the 4,4'-biphenyldicarboxylate $\left(\mathrm{bpdc}^{2-}\right)$ and Hf-containing analogue of UiO-66 (Figure 1). When a large excess of formic acid was used as a modulator in the synthesis of UiO-67, a new phase formed instead of the defect nanodomain phase observed with benzene-1,4-dicarboxylic acid. Structure solution from laboratory PXRD data revealed a hexagonal 3D MOF consisting of an hcp array of $\mathrm{Hf}_{12} \mathrm{O}_{8}(\mathrm{OH})_{14}$ clusters connected by bpdc ${ }^{2-}$ ligands (Figure $1 \mathrm{~b}, \mathrm{c})$. This $\mathrm{Hf}_{12}$ cluster consists of two face-sharing $\mathrm{Hf}_{6} \mathrm{O}_{4}(\mathrm{OH})_{4}$ clusters linked by six $\mu_{2}-\mathrm{OH}$ ligands (Figure 1f) and has to date been reported only as a molecular species. ${ }^{52,53}$ These six $\mu_{2}-\mathrm{OH}$ sites per double cluster are in addition to the eight $\mu_{3}-\mathrm{OH}$ ligands present in the two clusters (Figure 1f). The high acidity of bridging hydroxide groups in $\mathrm{Zr}$ and $\mathrm{Hf}$ MOFs has been previously noted, ${ }^{54,55}$ and the proximity of this plane of six $\mu_{2}-\mathrm{OH}$ ligands to the $\mu_{3}-\mathrm{OH}$ ligands suggests that this $\mathrm{Hf}_{12}$ cluster might show reactivity and catalytic behavior similar to that of the silanol nests found in defective zeolites. ${ }^{56}$

Each $\mathrm{Hf}_{6}$ cluster is bonded to only nine (rather than 12) bpdc $^{2-}$ ligands, and therefore, the ligand:metal ratio has been reduced from 12:2 to 9:2. The structure can be considered a layered polytype of fcu UiO-67, where the clusters adopt an "ABBA" stacking sequence rather than the typical "ABC" stacking found in fcu UiO-67 (Figure 1d,e). The relationship between hcp and fcu UiO-67 bears a striking resemblance to that in the mixed cubic/hexagonal oxide perovskites (ideal formula $\mathrm{ABO}_{3}$ ) (Figure 1g,h). In this family, structural variety results from the ability of the B-site octahedra to either cornershare or face-share. ${ }^{57,58}$ Where all of the octahedra cornershare, this leads to the cubic perovskite (" $3 C$ ") structure, analogous to fcu UiO-67. If the B-site octahedra alternate between corner- and face-sharing, the " $4 \mathrm{H}$ " structure results, analogous to hcp UiO-67.

Ligand substoichiometry in MOFs is ordinarily accommodated through ligand vacancies and hence leads to increased porosity, ${ }^{11}$ but in hcp UiO-67, as often occurs in oxides, cluster condensation produces a denser material. Despite this decrease in porosity, hcp UiO-67 remains microporous, with a Brunauer-Emmett-Teller surface area $\left(\mathrm{N}_{2}\right)$ of $1424 \mathrm{~m}^{2} \mathrm{~g}^{-1}$.
Simulation of the $\mathrm{N}_{2}$ isotherm using grand canonical Monte Carlo calculations on the determined crystal structure of hcp UiO-67 proved consistent with the experimentally observed isotherms (Supporting Discussion 1 and Supporting Figures 13).

To gain greater insight into the formation of this new phase, we carried out a series of experiments that included varying the synthetic conditions and carrying out in situ synchrotron PXRD measurements of the synthesis of hcp UiO-67. Ex situ investigation of the synthesis conditions revealed that the crucial factors for the formation of the hcp phase rather than the fcu phase are higher temperatures $\left(\geq 130{ }^{\circ} \mathrm{C}\right)$ and the presence of large quantities of formic acid as a modulator (Supporting Figure 4). With low quantities of formic acid, fcu UiO-67 forms, and at lower temperatures an unsolved and poorly crystalline material forms. The propensity of higher temperatures to promote the formation of higher-nuclearity metal oxide clusters is well-known for zirconium and hafnium MOFs. ${ }^{59}$ In situ synchrotron PXRD measurements carried out under the previously identified synthetic conditions allowed us to probe the evolution of the crystallization of this new phase through time (Figure 2). The use of formic acid as a modulator

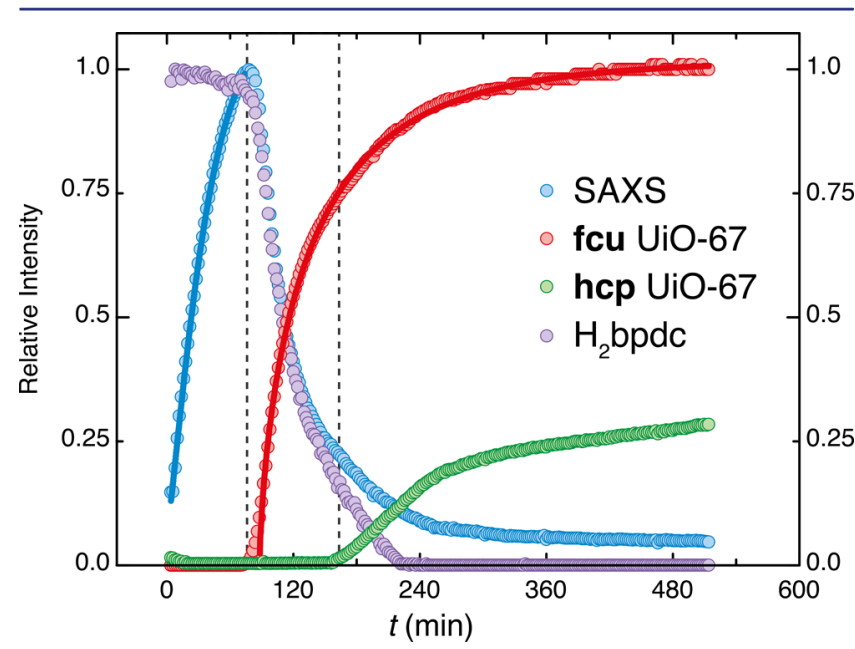

Figure 2. Evolution of phases during the synthesis of hcp UiO-67 synthesized at $150{ }^{\circ} \mathrm{C}$ over $8 \mathrm{~h}$. The intensity for each phase has been rescaled to lie between zero and one, with the fcu UiO-67 and hcp UiO-67 phases placed on the same scale (relative to $\mathrm{Hf}$ ) such that the final quantity of fcu UiO-67 is equal to 1 . The SAXS intensity was monitored by integrating the low-angle contribution above a fixed background, fcu UiO-67 through the intensity of the (111) reflection, hcp UiO-67 through the intensity of the (002) reflection, and $\mathrm{H}_{2}$ bpdc through the intensity of the most intense peak at $Q=1.36 \AA^{-1}$. Fits to the crystal growth of the fcu UiO-67 and SAXS intensities are shown as solid lines, and vertical dotted lines have been added to indicate the start of the growth of fcu and hcp UiO-67.

led to much slower crystallization than observed previously for fcu UiO-67 synthesized with $\mathrm{HCl}$ (formation time $t_{\mathrm{f}}=3$ $\min ),{ }^{60}$ so we were unable to probe in situ its crystallization to completion (the final hcp:fcu phase ratio was 0.31 ). The slow kinetics of this reaction are likely due, at least in part, to the low solubility of $\mathrm{H}_{2} \mathrm{bpdc}$ in formic acid/DMF solutions (as evidenced by the presence of ligand peaks in the diffraction data). This reduced ligand concentration, in combination with the competition with the high concentration of formic acid, likely explains the reduced ligand:metal ratio seen in hcp UiO67. The precise mechanism for the promotion of hcp UiO-67 
(a)

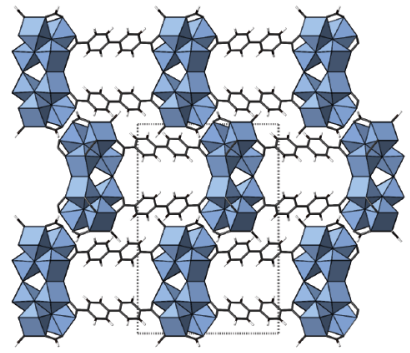

[110]

(d)

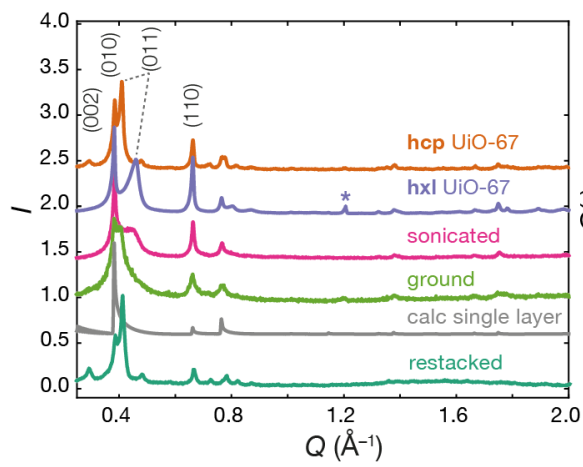

(b)

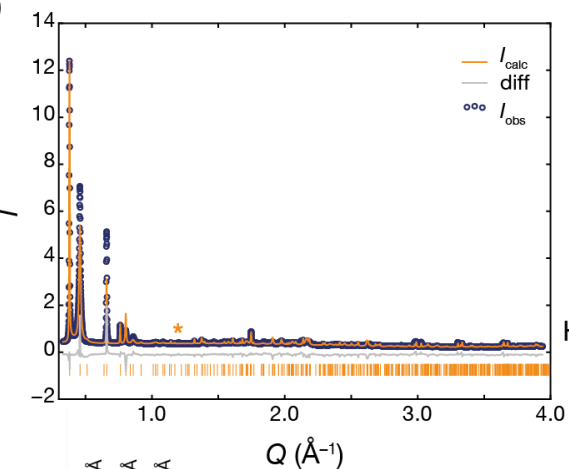

(c)

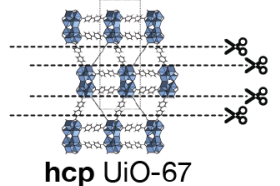

hcp UiO-67

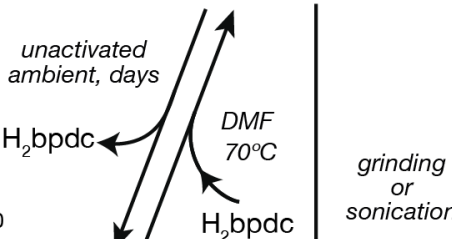

(e)
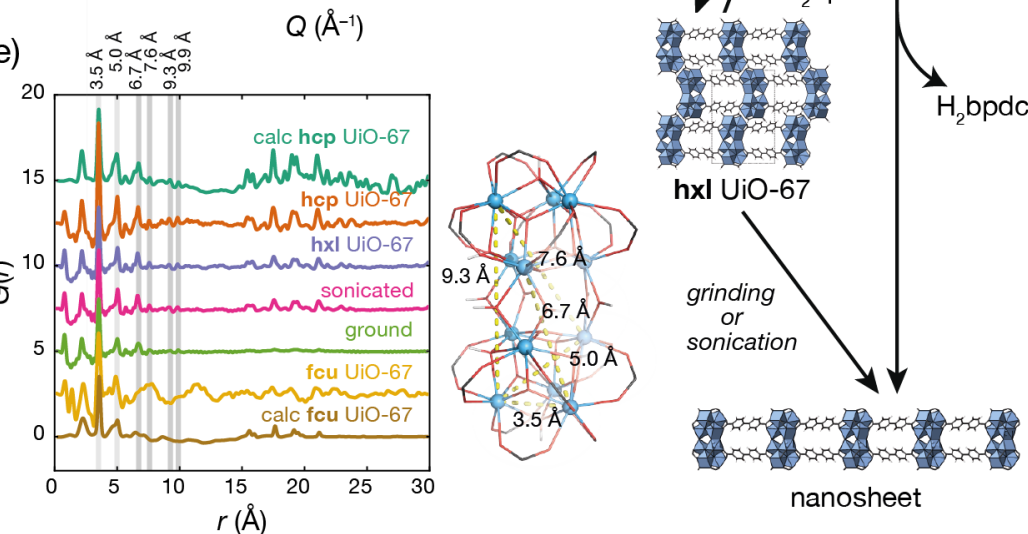

Figure 3. Low-dimensional structures formed from hcp UiO-67. (a) Structure of hxl UiO-67 in the polyhedral representation, viewed along the [100] axis. (b) Rietveld refinement of PXRD diffraction data for hxl UiO-67. Discrepancies in the low- $Q$ reflection intensities are likely due to disordered guests in pores. A peak due to the presence of ligand is highlighted with *. (c) Schematic illustration of the chemical transformations of hcp UiO-67. (d) PXRD patterns of the phases accessible from hcp UiO-67, compared with a calculated diffraction pattern from a single layer of double clusters. A peak due to the presence of ligand is highlighted with *. (e) PDFs of UiO-67-related phases, compared with calculated PDFs for hcp UiO-67 and fcu UiO-67. In all of the hcp UiO-67-derived phases, peaks corresponding to distances in the double cluster are present. Structures are represented as coordination polyhedra, with $\mathrm{HfO}_{8}$ polyhedra shown in blue. Color scheme: Hf, blue; O, red; $\mathrm{H}$, white; C, black.

by formic acid may be more complex, as formic acid can both influence the equilibrium of DMF hydrolysis ${ }^{61}$ and act as a $\mu_{2}$ ligand, which perhaps permits the formation of a transient formate-bridged double cluster precursor. ${ }^{22,62}$

The formation of hcp UiO-67 occurs under these conditions in three stages (Figure 2). First, a noncrystalline aggregate forms $\left(t_{\mathrm{f}}=40(2) \mathrm{min}\right)$, which is then rapidly consumed along with the remaining undissolved ligand as fcu UiO-67 forms. The increase in small-angle scattering $\left(Q<0.3 \AA^{-1}\right)$-the signature of this aggregation-is not accompanied by a significant decrease in the quantity of undissolved ligand, implying that this aggregate is purely inorganic. The formation of inorganic precursors is known for other MOF materials. ${ }^{63}$ Once fcu UiO-67 has begun to form (induction time $t_{\mathrm{i}}=$ 88.0(0.3) min, $t_{\mathrm{f}}=49.3(1.3) \mathrm{min}$ ) the hcp UiO-67 phase begins to crystallize. The crystallization of hcp UiO-67 proceeds unusually and does not follow classical nucleation-growth kinetics as modeled by either the Avrami or Gualtieri equations ${ }^{64,65}$ but instead continues at a roughly constant rate, suggesting that the precursor of the hcp UiO-67 phase is in large excess (Supporting Discussion 2, Supporting Figures 6-8, and Supporting Table 2). The structural changes during crystallization for both fcu and hcp UiO-67 are relatively subtle, with only small changes in the lattice parameters and peak width observed during crystallization, which suggests that crystal growth may be more rapid than new nucleation in this phase (Supporting Figure 8).

Although this defective phase is the more stable phase under the synthetic conditions, as-synthesized hcp UiO-67 is less robust than fcu UiO-67 after synthesis and transforms over a period of days under ambient conditions into a new phase, hxl UiO-67 (Figure 3). hxl UiO-67 was indexed from synchrotron PXRD data with a cell similar to that of hcp UiO-67, except for a radically contracted $c$ axis (reduction of 44\%) (Figure 3b). Solution of the structure from synchrotron PXRD data reveals that this transformed phase is very closely related to hcp UiO67 , but the bpdc ${ }^{2-}$ ligands that gave rise to the third dimension of organic connectivity have been lost, leaving hxl topology layers of double clusters connected by bpdc ${ }^{2-}$ ligands that stack in a staggered fashion (Figure 3a). These layers are not covalently bonded along the $c$ direction, which is consistent with the observed pronounced $h k l$-dependent peak broadening in the PXRD data, indicative of a dramatic reduction of order in the $c$ direction (correlation length or domain size of $40(5) \mathrm{nm}$ ). hxl UiO-67 does not transform further under ambient conditions and is stable (by PXRD) for more than a year. A small peak due to the crystalline ligand was observed in the powder diffraction data. We found that upon washing in hot DMF $\left(70{ }^{\circ} \mathrm{C}\right)$ this residual ligand could be recoordinated to the hxl sheets, reforming hcp UiO-67. The ease with which the $\mathrm{Hf}-$ carboxylate bonds that connect the sheets break and form suggested that it might be possible to delaminate both the hcp and hxl phases to produce isolated nanosheets.

After either extended grinding or sonication in $\mathrm{MeOH}$ of both hcp and hxl UiO-67, PXRD measurements showed the presence of only $(h k 0)$ reflections with pronounced "Warren"type line shapes (Figure 3d), ${ }^{66}$ indicative of the absence of long-range order in the stacking direction and the retention of 
periodicity within the plane (Figure 3e). For nanosheet samples generated through sonication, a broad feature is retained. This feature is consistent with a severely broadened (011) reflection of the hxl phase, with the broadening resulting from an approximate crystallite thickness or correlation length of 10(5) $\mathrm{nm}$. Calculated diffraction patterns from models of nanosheets of various thicknesses with differing terminations (including a layer of single clusters, a layer of double clusters, and a twolayer fragment of hcp UiO-67) were all broadly consistent with the experimental data but proved unable to categorically distinguish between the models (Supporting Figure 14). We therefore collected a number of X-ray PDFs on samples from the UiO-67 family. In all cases the presence of intact Hf clusters and intercluster connectivity within the $a b$ plane could be discerned, although the degree of intercluster order was much weaker for the poorly crystalline ground sample (Figure 3e). Comparison of the PDFs for the hcp UiO-67-derived samples with that of fcu UiO-67 shows the presence of additional peaks between 5.0 and $10.0 \AA$, characteristic of $\mathrm{Hf}-\mathrm{Hf}$ distances between the two $\mathrm{Hf}_{6}$ octahedra in the double cluster, showing that the nanosheets are formed from double clusters. This confirms that the $2 \mathrm{D} \mathrm{hxl}$ sheets are formed from hcp UiO-67 by breaking metal-bpdc ${ }^{2-}$ bonds rather than the cleavage of the $\mu_{2}-\mathrm{OH}$ bonds between the clusters.

The anisotropic chemical stability of hcp UiO-67 is consistent with the higher connectivity within the $a b$ plane (there are twice as many ligands within the plane as between planes). In order to assess whether there is also an energetic contribution to the selectivity of substitution, we carried out quantum-chemical calculations to determine the relative energy change of substitution for the replacement of one bpdc ${ }^{2-}$ ligand per unit cell by two formates for both interplane and intraplane ligand vacancies. This showed a significant energetic preference for removing an interplane ligand over a ligand within the $a b$ plane $\left(\Delta E_{c}-\Delta E_{a b}=+9.53 \mathrm{~kJ} \mathrm{~mol}^{-1}\right)$. This energetic preference continues at higher vacancy concentrations: for two ligand vacancies per cell, the energy difference per ligand between intralayer vacancies and interlayer vacancies becomes even more positive $\left(\Delta E_{2 c}-\Delta E_{a b+c}=+13.0 \mathrm{~kJ} \mathrm{~mol}^{-1}\right.$ per ligand; $\Delta E_{2 c}-\Delta E_{2 a b}=+17.9 \mathrm{~kJ} \mathrm{~mol}^{-1}$ per ligand). The introduction of a third interlayer vacancy per cell removes all threedimensional connectivity between two sheets (as would be the case in the 2D hxl UiO-67 phase). Relaxation of the cell reveals that these three interplane vacancies are strongly stabilized relative to three intraplane vacancies $\left(\Delta E_{3 c}-\Delta E_{3 a b}=+174.1 \mathrm{~kJ}\right.$ $\mathrm{mol}^{-1}$ per ligand). These calculations suggest that gradual substitution of the bpdc ${ }^{2-}$ ligand with monodentate capping ligands can proceed in a chemically selective manner and thus that the topotactic formation of hxl UiO-67 through this process is plausible. NMR analysis of digested hxl UiO-67 indeed showed the presence of a significant concentration of formate $\left(\mathrm{bpdc}^{2-}\right.$ :formate $=1: 0.10$; see Supporting Figure 15), indicating that it is likely present as one of the capping ligands for the hxl layers. The remainder of the capping ligands are likely to be hydroxide and water, which would not be detected in the NMR spectrum of the sample after digestion (1 M $\mathrm{NaOH}$ in $\mathrm{D}_{2} \mathrm{O}$ ) but have previously been shown to be present in defective UiO-type MOFs. ${ }^{23,67}$

HRTEM micrographs and SAED patterns confirmed that the individual layers retained their two-dimensional connectivity, showing a hexagonal arrangement with a spacing of 19(1) $\AA$ (HRTEM) and 19.0(5) A (SAED) (Figure 4). The beam sensitivity of the sample unfortunately precluded the collection
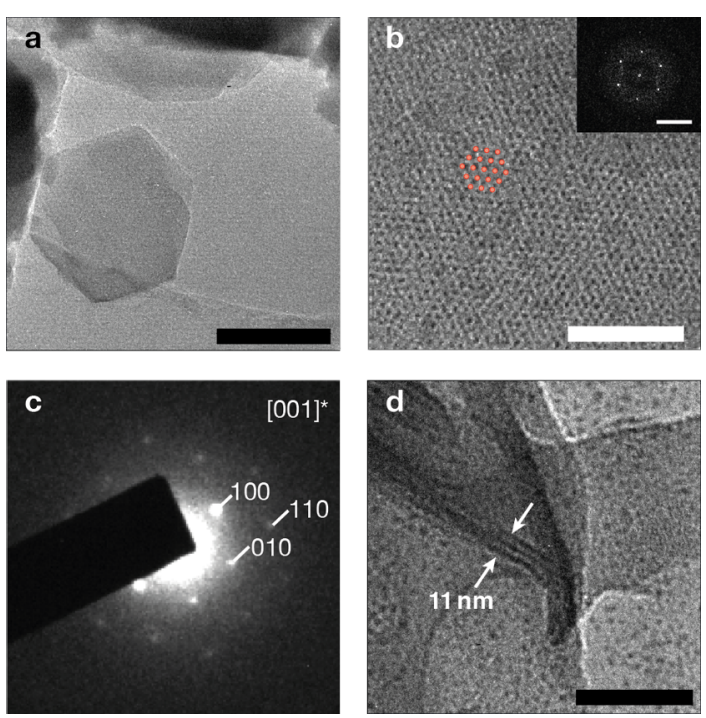

Figure 4. Micrographs of nanosheets of hxl UiO-67. (a) TEM micrograph of nanosheets, illustrating their hexagonal morphology (scale bar $=300 \mathrm{~nm}$ ). (b) HRTEM image of an hxl nanosheet (scale bar $=20 \mathrm{~nm}$ ). A hexagonal array of red circles with a $19 \AA$ A separation has been added for comparison. The inset shows the Fourier transform of the micrograph, highlighting the hexagonal symmetry (scale bar $=1$ $\mathrm{nm}^{-1}$ ). (c) SAED pattern of a nanosheet. (d) Measurement of curled sheet edges gives a thickness of 11(1) nm, equivalent to approximately four unit cells of hxl UiO-67 $(4 c=9.89 \mathrm{~nm})($ scale bar $=40 \mathrm{~nm})$.

of the very high resolution images or diffraction patterns necessary for structure determination or refinement. Examination of the edges of this material did however show that these sheets were $11(1) \mathrm{nm}$ thick, corresponding to approximately four unit cells of the transformed phase $(4 c=9.89 \mathrm{~nm}$ ) (Figure $4 \mathrm{~d}$ ), which was confirmed by AFM measurements (Supporting Figure 17).

\section{CONCLUSIONS}

In this work, we have explored the chemistry of a new member of the UiO family of MOFs: hcp UiO-67. This material exhibits a new structural mechanism for the accommodation of reduced ligand:metal ratios in MOFs, namely, cluster condensation. This mechanism also provokes analogies with the behavior of traditional inorganic materials, where the structural adaptability generated by varying stacking sequences can be a crucial parameter for the functional properties of a material, e.g., in the many families of layered perovskites ${ }^{57,58}$ or in the $\mathrm{ABO}_{2}$ transition metal oxides widely used in battery cathode materials. $^{68}$ That hcp UiO-67 and fcu UiO-67 possess an equivalent structural relationship suggests that there will be similar diversity available in MOF chemistry, as further supported by the recent report of edge-sharing clusters in a Zr-MOF. ${ }^{69}$

We have also demonstrated that the metal-ligand bonds in hcp UiO-67 are labile under appropriate conditions and can be selectively cleaved to form two-dimensional materials. The potential utility of specific chemical weaknesses in porous materials has already been noted, ${ }^{70}$ especially in the context of zeolites. $^{71}$ We have shown that it is possible to use defect engineering to introduce these "weak links" into even robust MOFs such as fcu UiO-67, and it is therefore likely that this strategy could be used to create other metal-organic nanosheets from 3D MOFs. Previous work on zeolites has 
shown that reassembling cleaved materials is a productive route to functional materials inaccessible through direct synthesis. ${ }^{70}$ The reversibility of the $\mathbf{h x l}-\mathbf{h} \mathbf{c p}$ transformation suggests that carrying out this reassembly with differently functionalized ligands, as a form of "postsynthetic" ligand exchange, ${ }^{72}$ could be used to create new families of 3D MOFs. This reassembly would provide control over the porosity of these $2 \mathrm{D}$ materials in the third dimension, as the choice of ligand will dictate not only the shape of the resultant pores but also their chemical functionality (e.g., hydrophobicity). This would therefore be a step toward more effective use of $2 \mathrm{D}$ materials in separation applications. ${ }^{73,74}$ The chemistry developed here with hcp UiO67 thus provides a platform for the creation of new kinds of metal-organic materials, both two- and three-dimensional.

\section{ASSOCIATED CONTENT}

\section{S Supporting Information}

The Supporting Information is available free of charge on the ACS Publications website at DOI: 10.1021/jacs.7b00106.

Calculated structural coordinates in CIF format (ZIP) Additional experimental details, including diffraction analyses, TGA data, adsorption experiments, and AFM data $(\mathrm{PDF})$

\section{AUTHOR INFORMATION}

\section{Corresponding Author}

*cpg27@cam.ac.uk

\section{ORCID}

Matthew J. Cliffe: 0000-0002-0408-7647

Elizabeth Castillo-Martínez: 0000-0002-8577-9572

Peyman Z. Moghadam: 0000-0002-1592-0139

\section{Notes}

The authors declare no competing financial interest.

\section{ACKNOWLEDGMENTS}

The authors thank Dr. Gregor Kieslich, Josh Stratford, Dr. Phoebe Allan, and Dr. Maria Tsiamtsouri for carrying out X-ray PDF measurements and Dr. David Jefferson for useful discussions. M.J.C. was supported by Sidney Sussex College, Cambridge; M.J.C., J.A.H., and A.L.G. were supported by the European Research Council (279705); and J.L., A.C.F., E.C.M., and C.P.G. were supported by the Engineering and Physical Sciences Research Council (U.K.) under the Supergen Consortium and Grant (EP/N001583/1). D.F.-J. thanks the Royal Society for funding through a University Research Fellowship. The Diamond Light Source Ltd. (beamlines I11 (EE9940, EE15118), I12 (EE12554), and I15 (EE13681, EE13843) is thanked for providing beamtime. Via our membership of the UK's HEC Materials Chemistry Consortium, which is funded by EPSRC (EP/L000202), this work used the ARCHER UK National Supercomputing Service (http://www.archer.ac.uk). Part of this work was performed using the Darwin Supercomputer of the University of Cambridge High Performance Computing Service (http:// www.hpc.cam.ac.uk/), provided by Dell Inc. using Strategic Research Infrastructure Funding from the Higher Education Funding Council for England and funding from the Science and Technology Facilities Council.

\section{REFERENCES}

(1) Dresselhaus, M. S.; Chen, G.; Tang, M. Y.; Yang, R.; Lee, H.; Wang, D.; Ren, Z.; Fleurial, J. P.; Gogna, P. Adv. Mater. 2007, 19, 1043-1053.

(2) Kieslich, G.; Cerretti, G.; Veremchuk, I.; Hermann, R. P.; Panthöfer, M.; Grin, J.; Tremel, W. Phys. Status Solidi A 2016, 213, 808-823.

(3) Andersson, S.; Sundholm, A.; Magnéli, A.; Högberg, B.; Kneip, P.; Palmstierna, H. Acta Chem. Scand. 1959, 13, 989-997.

(4) Allred, J. M.; Cava, R. J. J. Solid State Chem. 2013, 198, 10-17.

(5) Treacy, M. M. J.; Rice, S. B.; Jacobson, A. J.; Lewandowski, J. T. Chem. Mater. 1990, 2, 279-286.

(6) Ebina, Y.; Sasaki, T.; Harada, M.; Watanabe, M. Chem. Mater. 2002, 14, 4390-4395.

(7) Tong, Z.; Zhang, G.; Takagi, S.; Shimada, T.; Tachibana, H.; Inoue, H. Chem. Lett. 2005, 34, 632-633.

(8) Fang, Z.; Bueken, B.; De Vos, D. E.; Fischer, R. A. Angew. Chem., Int. Ed. 2015, 54, 7234-7254.

(9) Cheetham, A. K.; Bennett, T. D.; Coudert, F.-X.; Goodwin, A. L. Dalton Trans. 2016, 45, 4113-4126.

(10) Bunck, D. N.; Dichtel, W. R. Chem. - Eur. J. 2013, 19, 818-827.

(11) Wu, H.; Chua, Y. S.; Krungleviciute, V.; Tyagi, M.; Chen, P.; Yildirim, T.; Zhou, W. J. Am. Chem. Soc. 2013, 135, 10525-10532.

(12) Vermoortele, F.; Bueken, B.; Le Bars, G.; Van de Voorde, B.; Vandichel, M.; Houthoofd, K.; Vimont, A.; Daturi, M.; Waroquier, M.; Van Speybroeck, V.; Kirschhock, C.; De Vos, D. E. J. Am. Chem. Soc. 2013, 135, 11465-11468.

(13) Shearer, G. C.; Chavan, S.; Bordiga, S.; Svelle, S.; Olsbye, U.; Lillerud, K. P. Chem. Mater. 2016, 28, 3749-3761.

(14) Kozachuk, O.; Luz, I.; Llabrés I Xamena, F. X.; Noei, H.; Kauer, M.; Albada, H. B.; Bloch, E. D.; Marler, B.; Wang, Y.; Muhler, M.; Fischer, R. A. Angew. Chem., Int. Ed. 2014, 53, 7058-7062.

(15) Tsuruoka, T.; Furukawa, S.; Takashima, Y.; Yoshida, K.; Isoda, S.; Kitagawa, S. Angew. Chem., Int. Ed. 2009, 48, 4739-4743.

(16) Schaate, A.; Roy, P.; Godt, A.; Lippke, J.; Waltz, F.; Wiebcke, M.; Behrens, P. Chem. - Eur. J. 2011, 17, 6643-6651.

(17) Morris, W.; Volosskiy, B.; Demir, S.; Gándara, F.; McGrier, P. L.; Furukawa, H.; Cascio, D.; Stoddart, J. F.; Yaghi, O. M. Inorg. Chem. 2012, 51, 6443-6445.

(18) Feng, D.; Gu, Z. Y.; Li, J. R.; Jiang, H. L.; Wei, Z.; Zhou, H. C. Angew. Chem., Int. Ed. 2012, 51, 10307-10310.

(19) Mondloch, J. E.; Bury, W.; Fairen-Jimenez, D.; Kwon, S.; DeMarco, E. J.; Weston, M. H.; Sarjeant, A. A.; Nguyen, S. T.; Stair, P. C.; Snurr, R. Q.; Farha, O. K.; Hupp, J. T. J. Am. Chem. Soc. 2013, 135, 10294-10297.

(20) Ma, J.; Tran, L. D.; Matzger, A. J. Cryst. Growth Des. 2016, 16, $4148-4153$.

(21) O'Keeffe, M.; Peskov, M. A.; Ramsden, S. J.; Yaghi, O. M. Acc. Chem. Res. 2008, 41, 1782-1789.

(22) Cliffe, M. J.; Wan, W.; Zou, X.; Chater, P. A.; Kleppe, A. K.; Tucker, M. G.; Wilhelm, H.; Funnell, N. P.; Coudert, F.-X.; Goodwin, A. L. Nat. Commun. 2014, 5, 4176.

(23) Øien, S.; Wragg, D.; Reinsch, H.; Svelle, S.; Bordiga, S.; Lamberti, C.; Lillerud, K. P. Cryst. Growth Des. 2014, 14, 5370-5372.

(24) Nicolosi, V.; Chhowalla, M.; Kanatzidis, M. G.; Strano, M. S.; Coleman, J. N. Science 2013, 340, 1226419.

(25) Ramakrishna Matte, H. S. S.; Gomathi, A.; Manna, A. K.; Late, D. J.; Datta, R.; Pati, S. K.; Rao, C. N. R. Angew. Chem., Int. Ed. 2010, 49, 4059-4062.

(26) Liu, J.; Xue, Y.; Wang, Z.; Xu, Z.-Q.; Zheng, C.; Weber, B.; Song, J.; Wang, Y.; Lu, Y.; Zhang, Y.; Bao, Q. ACS Nano 2016, 10, $3536-3542$

(27) Varoon, K.; Zhang, X.; Elyassi, B.; Brewer, D. D.; Gettel, M.; Kumar, S.; Lee, J. A.; Maheshwari, S.; Mittal, A.; Sung, C.-Y.; Cococcioni, M.; Francis, L. F.; McCormick, A. V.; Mkhoyan, K. A.; Tsapatsis, M. Science 2011, 334, 72-75.

(28) Chen, W.-F.; Sasaki, K.; Ma, C.; Frenkel, A. I.; Marinkovic, N.; Muckerman, J. T.; Zhu, Y.; Adzic, R. R. Angew. Chem., Int. Ed. 2012, $51,6131-6135$. 
(29) Choi, M.; Na, K.; Kim, J.; Sakamoto, Y.; Terasaki, O.; Ryoo, R. Nature 2009, 461, 828-828.

(30) Maeda, H.; Sakamoto, R.; Nishihara, H. Langmuir 2016, 32, $2527-2538$.

(31) Rodenas, T.; Luz, I.; Prieto, G.; Seoane, B.; Miro, H.; Corma, A.; Kapteijn, F.; Llabrés i Xamena, F. X.; Gascon, J. Nat. Mater. 2014, 14, $48-55$.

(32) Cao, L.; Lin, Z.; Peng, F.; Wang, W.; Huang, R.; Wang, C.; Yan, J.; Liang, J.; Zhang, Z.; Zhang, T.; Long, L.; Sun, J.; Lin, W. Angew. Chem., Int. Ed. 2016, 55, 4962-4966.

(33) Zhao, M.; Lu, Q.; Ma, Q.; Zhang, H. Small Methods 2017, 1, 1600030 .

(34) Makiura, R.; Konovalov, O. Sci. Rep. 2013, 3, 2506.

(35) Foster, J. A.; Henke, S.; Schneemann, A.; Fischer, R. A.; Cheetham, A. K. Chem. Commun. 2016, 52, 10474-10477.

(36) Zhao, M.; Wang, Y.; Ma, Q.; Huang, Y.; Zhang, X.; Ping, J.; Zhang, Z.; Lu, Q.; Yu, Y.; Xu, H.; Zhao, Y.; Zhang, H. Adv. Mater. 2015, 27, 7372-7378.

(37) Thompson, S. P.; Parker, J. E.; Marchal, J.; Potter, J.; Birt, A.; Yuan, F.; Fearn, R. D.; Lennie, A. R.; Street, S. R.; Tang, C. C. J. Synchrotron Radiat. 2011, 18, 637-648.

(38) Thompson, S. P.; Parker, J. E.; Potter, J.; Hill, T. P.; Birt, A.; Cobb, T. M.; Yuan, F.; Tang, C. C. Rev. Sci. Instrum. 2009, 80, 075107.

(39) Pawley, G. S. J. Appl. Crystallogr. 1981, 14, 357-361.

(40) Rietveld, H. J. J. Appl. Crystallogr. 1969, 2, 65-71.

(41) Coelho, A. TOPAS-Academic: General Profile and Structure Analysis Software for Powder Diffraction Data, version 4.1; Coelho Software: Brisbane, Australia, 2007.

(42) Stokes, H. T.; Hatch, D. M.; Campbell, B. J. ISOTROPY Software Suite; Brigham Young University: Provo, UT; http://iso.byu. edu.

(43) Drakopoulos, M.; Connolley, T.; Reinhard, C.; Atwood, R.; Magdysyuk, O.; Vo, N.; Hart, M.; Connor, L.; Humphreys, B.; Howell, G.; Davies, S.; Hill, T.; Wilkin, G.; Pedersen, U.; Foster, A.; De Maio, N.; Basham, M.; Yuan, F.; Wanelik, K. J. Synchrotron Radiat. 2015, 22, 828-838.

(44) Moorhouse, S. J.; Vranješ, N.; Jupe, A.; Drakopoulos, M.; O'Hare, D. Rev. Sci. Instrum. 2012, 83, 084101.

(45) Basham, M.; Filik, J.; Wharmby, M. T.; Chang, P. C. Y.; El Kassaby, B.; Gerring, M.; Aishima, J.; Levik, K.; Pulford, B. C. A.; Sikharulidze, I.; Sneddon, D.; Webber, M.; Dhesi, S. S.; Maccherozzi, F.; Svensson, O.; Brockhauser, S.; Náray, G.; Ashton, A. W. J. Synchrotron Radiat. 2015, 22, 853-858.

(46) Juhás, P.; Davis, T.; Farrow, C. L.; Billinge, S. J. L. J. Appl. Crystallogr. 2013, 46, 560-566.

(47) McLain, S. E.; Bowron, D. T.; Hannon, A. C.; Soper, A. K. GudrunN and GudrunX: Programs for Correcting Raw Neutron and XRay Diffraction Data to Differential Scattering Cross Section; Rutherford Appleton Laboratory: Didcot, U.K., 2011.

(48) Clark, S. J.; Segall, M. D.; Pickard, C. J.; Hasnip, P. J.; Probert, M. I. J.; Refson, K.; Payne, M. C. Z. Kristallogr. - Cryst. Mater. 2005, 220, 567-570.

(49) Perdew, J. P.; Burke, K.; Ernzerhof, M. Phys. Rev. Lett. 1996, 77, 3865-3868.

(50) VandeVondele, J.; Krack, M.; Mohamed, F.; Parrinello, M.; Chassaing, T.; Hutter, J. Comput. Phys. Commun. 2005, 167, 103-128.

(51) VandeVondele, J.; Hutter, J. J. Chem. Phys. 2007, 127, 114105.

(52) Piszczek, P.; Radtke, A.; Wojtczak, A.; Muziol, T.; Chojnacki, J. Polyhedron 2009, 28, 279-285.

(53) Malaestean, I. L.; Speldrich, M.; Ellern, A.; Baca, S. G.; Kögerler, P. Dalton Trans. 2011, 40, 331-333.

(54) Ling, S.; Slater, B. Chem. Sci. 2016, 7, 4706-4712.

(55) Klet, R. C.; Liu, Y.; Wang, T. C.; Hupp, J. T.; Farha, O. K. J. Mater. Chem. A 2016, 4, 1479-1485.

(56) Dědeček, J.; Sklenak, S.; Li, C.; Gao, F.; Brus, J.; Zhu, Q.; Tatsumi, T. J. Phys. Chem. C 2009, 113, 14454-14466.

(57) Aleksandrov, K. S.; Beznosikov, V. V. Phys. Solid State 1997, 39, 695-715.
(58) Tilley, R. J. D. Perovskites: Structure-Property Relationships; John Wiley \& Sons: Chichester, U.K., 2016; Chapter 3, pp 79-122.

(59) Guillerm, V.; Ragon, F.; Dan-Hardi, M.; Devic, T.; Vishnuvarthan, M.; Campo, B.; Vimont, A.; Clet, G.; Yang, Q.; Maurin, G.; Férey, G.; Vittadini, A.; Gross, S.; Serre, C. Angew. Chem., Int. Ed. 2012, 51, 9267-9271.

(60) Ragon, F.; Chevreau, H.; Devic, T.; Serre, C.; Horcajada, P. Chem. - Eur. J. 2015, 21, 7135-7143.

(61) Wißmann, G.; Schaate, A.; Lilienthal, S.; Bremer, I.; Schneider, A. M.; Behrens, P. Microporous Mesoporous Mater. 2012, 152, 64-70.

(62) Liang, W.; Babarao, R.; Murphy, M. J.; D’Alessandro, D. M. Dalton Trans. 2015, 44, 1516-1519.

(63) Zheng, C.; Greer, H. F.; Chiang, C.-Y.; Zhou, W. CrystEngComm 2014, 16, 1064-1070.

(64) Gualtieri, A. F. Phys. Chem. Miner. 2001, 28, 719-728.

(65) Yeung, H. H.-M.; Wu, Y.; Henke, S.; Cheetham, A. K.; O'Hare, D.; Walton, R. I. Angew. Chem., Int. Ed. 2016, 55, 2012-2016.

(66) Warren, B. E. Phys. Rev. 1941, 59, 693-698.

(67) Vandichel, M.; Hajek, J.; Vermoortele, F.; Waroquier, M.; De Vos, D. E.; Van Speybroeck, V. CrystEngComm 2015, 17, 395-406.

(68) Delmas, C.; Fouassier, C.; Hagenmuller, P. Physica B+C 1980, 99, 81-85.

(69) Waitschat, S.; Reinsch, H.; Stock, N. Chem. Commun. 2016, 52, 12698-12701.

(70) Morris, R. E.; Čejka, J. Nat. Chem. 2015, 7, 381-388.

(71) Roth, W. J.; Nachtigall, P.; Morris, R. E.; Wheatley, P. S.; Seymour, V. R.; Ashbrook, S. E.; Chlubná, P.; Grajciar, L.; Položij, M.; Zukal, A.; Shvets, O.; Cejka, J. Nat. Chem. 2013, 5, 628-633.

(72) Kim, M.; Cahill, J. F.; Su, Y.; Prather, K. A.; Cohen, S. M. Chem. Sci. 2012, 3, 126-130.

(73) Lee, S. H.; Kim, H. W.; Hwang, J. O.; Lee, W. J.; Kwon, J.; Bielawski, C. W.; Ruoff, R. S.; Kim, S. O. Angew. Chem., Int. Ed. 2010, 49, 10084-10088.

(74) Bai, S.; Liu, X.; Zhu, K.; Wu, S.; Zhou, H. Nat. Energy 2016, 1, 16094. 